\title{
Q Fever in Pregnancy-Case Presentation and Literature Review
}

\author{
Kugler Eitan $^{1 *}$, Amital Howard ${ }^{2}$, Alon Danny ${ }^{3}$ \\ ${ }^{1}$ Rabin Medical Center, Beilinson Hospital, Department of Medicine F, Petah Tikva, Israel; ${ }^{2}$ Sheba Medical Center, Department of \\ Medicine B, Tel Hashomer, Israel; ${ }^{3}$ Rabin Medical Center, Beilinson Hospital, Department of Medicine B, Petah Tikva, Israel. \\ Email: *eitankugler@gmail.com
}

Received August $2^{\text {nd }}, 2012$; revised August $10^{\text {th }}, 2012$; accepted September $3^{\text {rd }}, 2012$

Copyright (c) 2013 Kugler Eitan et al. This is an open access article distributed under the Creative Commons Attribution License, which permits unrestricted use, distribution, and reproduction in any medium, provided the original work is properly cited.

\begin{abstract}
Q fever is a widespread zoonotic infection caused by Coxiella burnetii. During acute infection, three clinical patterns are commonly encountered—self limited, flu like disease, atypical pneumonia or hepatits. Chronic infection almost always implies endocarditis. Acute infection during pregnancy may result in obstetrical complications, and predispose to chronic transformation. Treatment during pregnancy is more complex, and the duration of treatment was debatable up until recently. We describe a 31 years old patient with acute $Q$ fever infection during pregnancy. We review the medical treatment, complications and follow up of acute and chronic $\mathrm{Q}$ fever during pregnancy.
\end{abstract}

Keywords: Q Fever; Coxiella burnetii; Pregnancy

\section{Introduction}

A 31 years old female with no significant medical history, presented to our department at 15 weeks of gestation, with a 10 days history of headaches, general muscle weakness, malaise with fever reaching 39 degrees.

As a tourist guide she reported frequent visits of caves and drinking unpasteurized milk, the last occurrence was two weeks prior to this hospitalization. It should be noted that her husband who shared the same occupation suffered from the similar symptoms.

On admission, her clinical and gynecological examination was unrevealing apart from a 2/6 systolic murmur over the aortic valve. Her laboratory results demonstrated normal white blood cell count with mild atypical lymphocytes and band forms appearing in her peripheral blood smear. The platelet count was normal. In addition, hepatocelluler liver enzymes were elevated up to 7 - 10 times the normal levels.

Due to the patients' medical history, a high suspicion of a zoonotic infection was taken. Serological testing was consistent with acute Q fever infection. Transthoracic echocardiography (TTE) revealed normal valvular morphology and function. Treatment with Cotrimoxazole was initiated.

During treatment, a widespread skin eruption appeared,

*Corresponding author. and treatment with azythromycin was initiated in place of cotrimoxazole.

Despite appropriate antibiotic treatment, serologic profile was consistent with chronic Q fever infection-phase 1 IgG titer was over 25,000 at week 37 of gestation. Labor was induced in order to start standard combination antibiotic treatment. A healthy baby girl was born, and Treatment with doxyciline and plaquenil was initiated immediately.

Figures 1-3 present the pathological findings of the patients’ placenta.

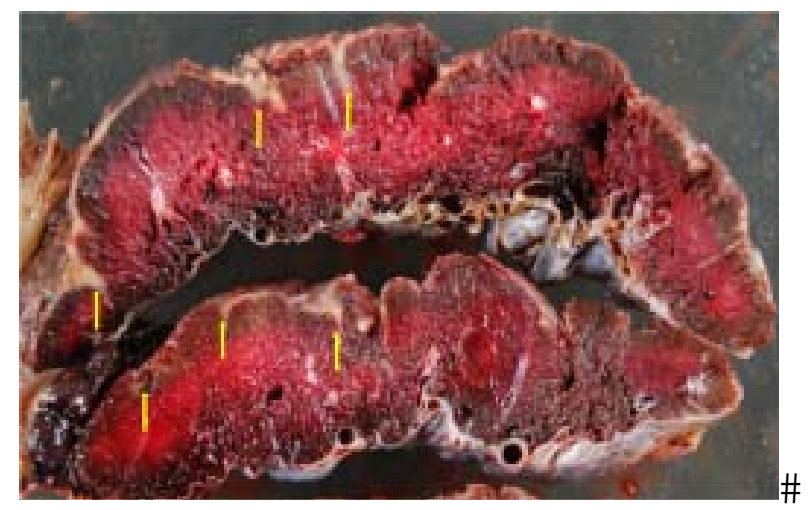

Figure 1. Gross view of placenta. Multiple foci of firm white tissue, flat or wedge shape (infract-like), involving maternal floor of placenta (yellow arrows). 


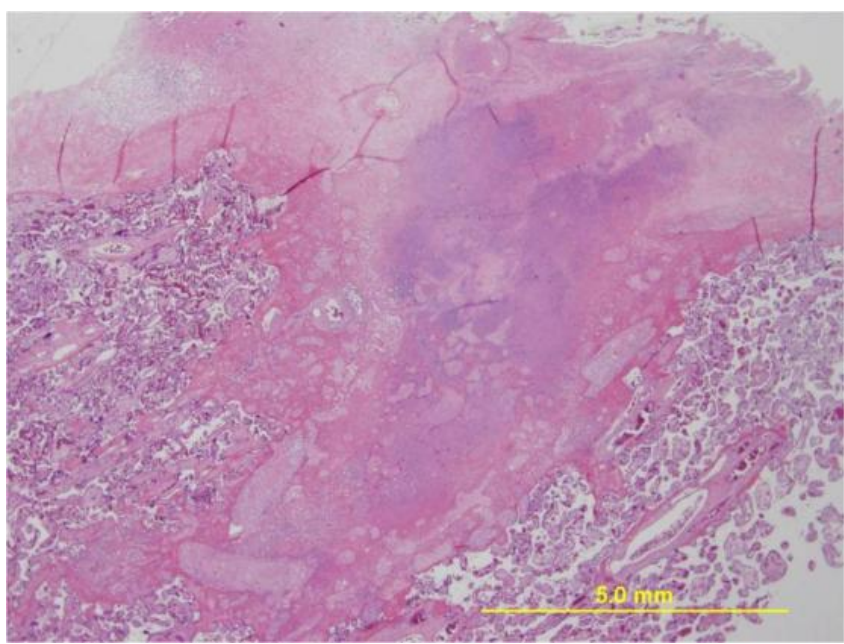

Figure 2. Histologic section of a wedge shaped focus: necrosis with abundant nuclear debris, surrounded with uninvolved placental villi $(\mathrm{H} \& \mathrm{E}, \times \mathbf{1 0 0})$.

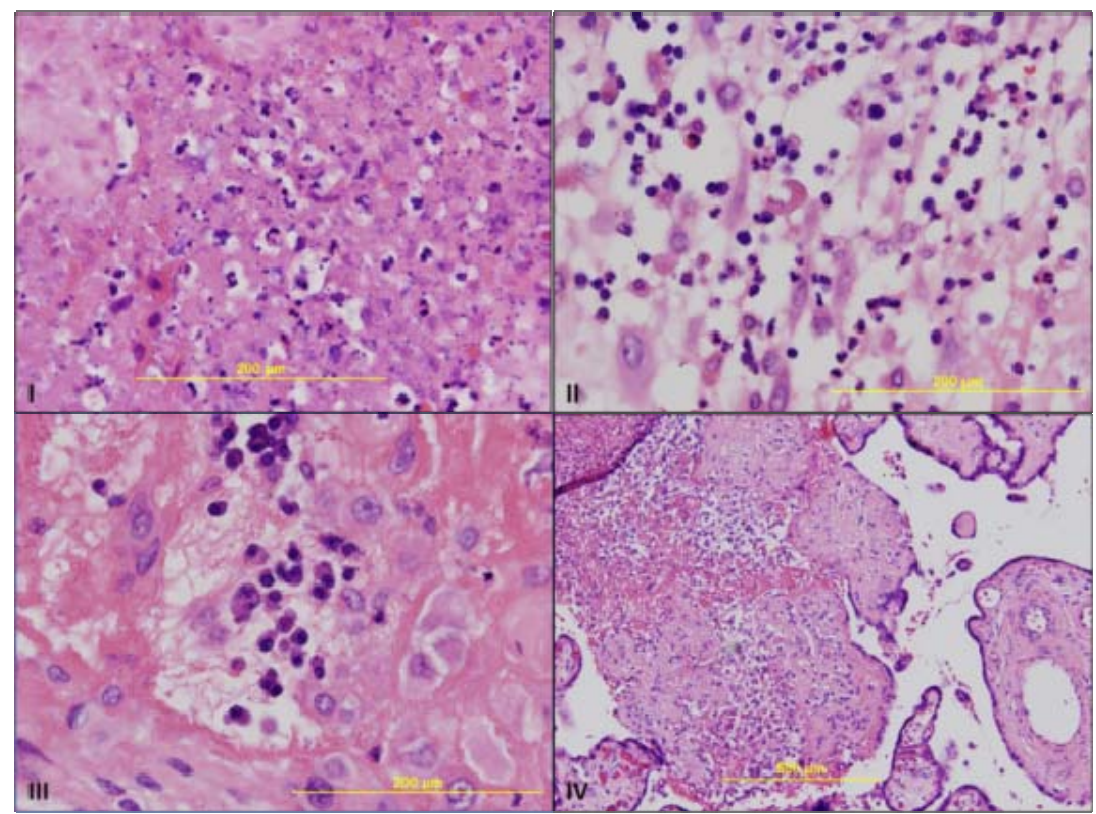

Figure 3. Details of involved maternal floor: (I) Necrosis intermixed with numerous disintegrating inflammatory cells in decidua (×100); (II) Polymorphonuclear leukocites in decidua (×100); (III) Plasma cells in decidua ( $\times 100)$; (IV) Necrotizing villitis and perivillitis (H\&E, ×50).

\section{Discussion}

Q fever is a widespread zoonotic infection caused by Coxiella burnetii (C. burnetii). While previously classified as a Rickettsia, C. burnetii has been placed into a subdivision of the Proteobacteria.

C. burnetii is a strict intracellular bacterium which can survive efficiently for a long period of time in highly acidic conditions such as the phagolysosomes of macrophages, or in the external environment. Sources of human infection are mainly cattle, sheep and goats. Infection follows inhalation of aerosolized organisms from infected parturient fluids. Occupational exposure is the most common form of acquisition. Consumption of raw milk is another source of infection.

C. burnetii was first described by Derrick in 1937 as a cause of both acute and chronic manifestations [1].

The majority of acute $\mathrm{Q}$ fever cases remain asymptomatic. Only $2 \%$ of patients with acute Q fever are hospitalized [2].

During acute infection, three clinical patterns are commonly encountered-a self-limitedshort "flu like" illness associated with headaches, atypical pneumonia and/or hepatits.

Pulmonary involvement is usually atypical in nature 
and most cases are mild, presenting with a non-productive cough, fever, and minimal auscultatory abnormalities. However, some patients present with acute respiratory distress. Pleural effusion can occur. Radiographic findings are usually non-specific, and resemble viral pneumonia. Symptoms can last from 10 to 90 days [2,3].

During acute Q fever hepatitis, serum transaminases are usually mildly elevated. Abdominal pain and jaundice are uncommon. Extensive destruction of the liver and hepatic failure was described [2]. Liver histology typically reveals a granulomatous hepatitis, even in asymptomatic patients. The hepatic lesion is very specific, in which some of the granulomas appear to be "doughnutlike" because they contain a lipidic vacuole surrounded by a fibrinoid ring. Moreover, patients with hepatits may exhibit autoantibodies directed to smooth muscle, anticardiolipin, anticoagulant and antinuclear antibodies [2].

The clinical presentation may vary, depending on the geographic location. Pneumonia is the major clinical presentation in Nova Scotia, Canada, in the Basque region in Spain and in Switzerland. Hepatits is more common in Ontario, Canada, in Andalusia, Spain, and in California, USA [2]. Animal models suggested that the route of inoculation, aerosol or ingestion of contaminated food (mostly raw milk) might be a proposed pathophysiologic mechanism for this phenomenon. Different strain of the pathogen may also be an explanation [2]. Raoult et al., described the clinical and epidemiologic feature of 1383 Q fever infections during 1985-1998, and has suggested that in any given geographic area, mostly host factors contribute to the clinical presentation during acute infection and not the route of inoculation. Patients with heaptitis were younger, less frequently immunocompromised, had more febrile episodes, more headache and myalgia, more thrombocytopenia and more elevated erythrocyte sedimentation rates. Patients who presented mainly with pulmonary involvement had the opposite epidemiologic and clinical characteristic. They were older, more immunocompromised, had less febrile episodes, suffered less headaches and myalgia, had more electrocardiogrphic abnormalities, and were less likely to develop thrombocytopenia. In addition, patients without pulmonary involvement or hepatitis were more frequently female [2].

Although acute Q fever infection is usually a mild and self limited disease, severe and rare complication can occur in approximately $1 \%$ of cases, and include myocarditis, pericarditis and meningoencephalitis. The pathogenesis is unknown, and patients from all age groups with no remarkable medical history may be involved. Myocarditis is probably underestimated, and is considered to be the most serious complication of acute $\mathrm{Q}$ fever infection [2].

Chronic infection is defined as infection lasting more than six months and it almost always implies endocardi- tis, though ostemyelitis and infection of vascular graft and aneurysms may occur. Occasionally, chronic hepatits in association with endocarditis or as an isolated condition may develop. Seldom, chronic infection can develop insidiously, months or years after the acute disease. Host factors play a role in the clinical expression of the disease and existence of an immunocompromised state, preexisting valvular heart disease or pregnancy predispose to chronic infection [2].

Treatment of acute Q fever is warranted only for symptomatic patients. Doxycycline $100 \mathrm{mg}$ twice daily for 2 weeks is the recommended regimen. Fluoroquinolones are useful as well, but are contraindicated in children and pregnant women. Prolonged treatment for a minimum of 18 months with doxycycline in combination with hydroxychloroquine is necessary in chronic infection.

All patients with acute Q fever should undergo transthoracic echocardiography to exclude the possibility of valvular heart disease, which mandates a longer treatment in order to prevent the progression to $\mathrm{Q}$ fever endocarditis. Therapy with doxycycline and hydroxychloroquine for a minimum of 12 months is warranted in this case [4].

Acute or chronic infection during pregnancy may result in obstetrical complication such as spontaneous abortion, intrauterine growth retardation, intrauterine fetal death, oligoamnios, and premature delivery. It appears that the risk is higher during the first trimester and that the majority of infected patients were asymptomatic $[5,6]$. Obstetrical adverse outcomes are frequent, occurring in up to $81 \%$ of infected patients [7].

Treating Q fever during pregnancy is more complex since doxycycline, fluoroquinollones and hydroxychloroquine are contraindicated. Cotrimoxazole (trimethoprim $320 \mathrm{mg} / \mathrm{sulphamethoxazole} 1600 \mathrm{mg}$ daily) has been found to be safe and effective. Up until recently, the duration of treatment was debatable. In 2007, a retrospective study of 53 pregnant patients with $\mathrm{Q}$ fever concluded that a long-term duration of cotrimoxazole protects against maternal transformation into chronic Q fever infection, placental infection, and obstetric complications regardless of gestational age. Therefore it is recommended from diagnosis until term [7].

In non-pregnant patients, the chance developing chronic $Q$ fever if a valvular abnormality exists is approximately $40 \%$ [4]. The chance to progress to chronic Q fever in pregnancy may reach $50 \%$ even if no valvular abnormality is present [7]. This may be due to high degree of bacterial colonization and replication in the maternal uterus and placenta, and to lack of appropriate immune response during pregnancy [5]. As mentioned earlier, those infected during the first trimester are at greater risk [7]. 
In addition, it should be noted that cotrimoxazole exerts a bacteriostatic effect on C. burnetii. As a result, it is recommended that all $\mathrm{Q}$ fever pregnant patients should be monitored serologically for a minimum of 24 months. Those with persisting Phase 1 IgG titers $>800$ on indirect fluorescent antibody assay should undergo transoesophageal echocardiography to detect early endocarditis. Treatment with doxycycline and hydroxychloroquine for a minimum of 18 months is indicated if endocarditis is confirmed [5].

Following delivery, a few precautions should be taken into account. C. burnetii may colonize breast milk, thus nursing should be avoided [8,9]. Furthermore, intrauterine transmission of $C$. burnetii has been documented in the past and in addition, an infected mother can potentially transmit the pathogen during delivery through aerosolized amniotic fluid thus it may be prudent to follow infants closely [10-12].

During treatment in this case, a wide spread skin eruption appeared, and treatment with azythromycin was initiated in place of cotrimoxazole.

Macrolides may represent a potential alternative for treatment of $\mathrm{Q}$ fever infection in children and pregnant women.

Erythromycin is now rarely used due to a small risk of sudden cardiac death due to QT interval prolongation in susceptible patients (acquired long QT syndrome or patient receiving other drugs concurrently which are metabolized by CYP3A4) [13].

Newer macrolides are more potent in vitro than erythromycin[14] and Preliminary clinical studies showed that they might be of clinical use [15].

In addition, skin rash may appear in $\mathrm{Q}$ fever infection in up to $10 \%-21 \%$ of cases. The eruption is not specific and may consist of maculopapular or purpuric rash of the trunk [2]. In the case presented here, it seemed likely to be due to drug allergy owing to a resolution upon drug replacement.

\section{Conclusions}

We presented a case of $\mathrm{Q}$ fever infection in a 31 years old patient during her second trimester of pregnancy. TTE was normal and treatment with cotrimoxazole was initiated. Later on it was switched to azythromycin due to skin eruption most probably related to drug allergy. Nevertheless, progression to chronic infection was evident by serology. Eventually labor was induced at week 37 due to rising titres, and treatment with doxyciline and plaquenil was initiated after delivery with decreasing antibody titres on follow up.

Q fever infection during pregnancy is a serious condition due to high rate of obstetrical complications and transformation to a chronic state of infection. Cotrimoxazole for the entire length of pregnancy, regardless of ge- stational age, should be administrated, while close follow up for serologic conversion should be mandatory for at least 24 months after delivery. In case of transformation to a chronic state, treatment with doxyciline and plaqeunil should be initiated immediately after delivery for 18 months. A strict gynecological observation must be made to rule out obstetrical complications. During labor, precautions must be carried out to prevent healthcare workers infection. Nursing should be avoided and serologic monitoring of the infant is required due to concern about aerosol or transplacental transmission.

\section{REFERENCES}

[1] E. H. Derrick, “'Q’ Fever, a New Fever Entity: Clinical Features, Diagnosis and Laboratory Investigation,” $\mathrm{Me}$ dical Journal of Australia, Vol. 2, 1937, pp. 281-229.

[2] D. Raoult, et al., "Q Fever 1985-1998. Clinical and Epidemiologic Features of 1383 Infections,” Medicine (Baltimore), Vol. 79, No. 2, 2000, pp. 109-123. doi:10.1097/00005792-200003000-00005

[3] D. Raoult and T. Marrie, "Q Fever," Clinical Infectious Diseases, Vol. 20, No. 3, 1995, p. 489. doi:10.1093/clinids/20.3.489

[4] F. Fenollar, P. E. Fournier, M. P. Carrieri, G. Habib, T. Messana and D. Raoult, "Risks Factors and Prevention of Q Fever Endocarditis,” Clinical Infectious Diseases, Vol. 33, No. 3, 2001, pp. 312-316. doi:10.1086/321889

[5] D. Raoult and F. Fenollar, "Stein A. Q Fever during Pregnancy: Diagnosis, Treatment, and Follow-Up,” Archives of Internal Medicine, Vol. 162, No. 6, 2002, pp. 701-704. doi:10.1001/archinte.162.6.701

[6] H. Tissot-Dupont, V. Vaillant, S. Rey and D. Raoult, "Role of Sex, Age, Previous Valve Lesion, and Pregnancy in the Clinical Expression and Outcome of Q Fever after a Large Outbreak," Clinical Infectious Diseases, Vol. 44, No. 2, 2007, pp. 232-237. doi:10.1086/510389

[7] X. Carcopino, D. Raoult, F. Bretelle, L. Boubli and A. Stein, "Managing Q Fever during Pregnancy: The Benefits of Long-Term Cotrimoxazole Therapy," Clinical Infectious Diseases, Vol. 45, No. 5, 2007, pp. 548-555. doi:10.1086/520661

[8] A. Kumar, M. P. Yadav and S. Kakkar, "Human Milk as a Source of Q-Fever Infection in Breast-Fed Babies,” Indian Journal of Medical Research, Vol. 73, 1981, pp. 510-512.

[9] B. N. Prasad, N. K. Chandiramani and A. Wagle, "Isolation of Coxiellaburnetii from Human Sources," International Journal of Zoonoses, Vol. 13, No. 2, 1986, pp. 112 117.

[10] A. Stein and D. Raoult, "Q Fever during Pregnancy: A Public Health Problem in Southern France," Clinical Infectious Diseases, Vol. 27, No. 3, 1998, pp. 592-596. doi:10.1086/514698

[11] D. Raoult and D. Stein, "Q Fever during Pregnancy: A Risk for Women, Fetuses, and Obstetricians [Letter]," The New England Journal of Medicine, Vol. 330, No. 5, 
1994, p. 371. doi:10.1056/NEJM199402033300518

[12] J. Denman and M. Woods, "Acute Q Fever in Pregnancy: Report and Literature Review," Internal Medicine Journal, Vol. 39, 2009, pp. 479-484.

doi:10.1111/j.1445-5994.2009.01933.x

[13] W. A. Ray, et al., "Oral Erythromycin and the Risk of Sudden Death from Cardiac Causes,” The New England Journal of Medicine, Vol. 351, No. 11, 2004, p. 1089. doi:10.1056/NEJMoa040582
[14] A. Boulos, et al., "Measurement of the Antibiotic Susceptibility of Coxiella burnetii Using Real Time PCR," International Journal of Antimicrobial Agents, Vol. 23, No. 2, 2004, p. 169. doi:10.1016/j.ijantimicag.2003.07.007

[15] A. Gikas, et al., "Newer Macrolides as Empiric Treatment for Acute Q Fever Infection,” Antimicrobial Agents and Chemotherapy, Vol. 45, No. 12, 2001, p. 3644. doi:10.1128/AAC.45.12.3644-3646.2001 